\title{
Click Chemistry: Mechanistic Insights into the role of amines using Single Molecule Spectroscopy
}

\author{
Bowen Wang, Anabel E. Lanterna* and Juan C. Scaiano* \\ Department of Chemistry and Biomolecular Sciences and Centre for Advanced Materials Research (CAMaR). Universi- \\ ty of Ottawa, 10 Marie Curie, Ottawa, ON. K1N 6N5 Canada.
}

KEYWORDS click chemistry, photocatalysis, single molecule spectroscopy, mechanisms

\begin{abstract}
Single molecule spectroscopy is used to gain insights into the click chemistry reaction photo-catalyzed by Copper. We show that $\mathrm{CuO}_{\mathrm{x}} @ \mathrm{Nb}_{2} \mathrm{O}_{5}$ catalysts can act as truly heterogeneous photocatalysts and that the amine plays an important role in the complexation between the alkyne and the copper active site on the catalyst surface. For complex reactions occurring in the sub-nanomolar range, pre-association can be essential and co-catalysts (such as amines here) may play an enabling role facilitating the reagents 'reunion' and prolonging the time available for reaction. This is to the best of our knowledge the first time the amine role is rationalized in heterogeneous photocatalytic click chemistry.
\end{abstract}

\section{INTRODUCTION}

The azide-alkyne cycloadditions have been amply studied since the first reports on the regioselective copper (I) catalysis (CuAAC) by Sharpless ${ }^{1}$ and Meldal $^{2}$ in 2002 (scheme 1). The reaction has been explored in both homogeneous and heterogeneous systems, where the stabilization of the $\mathrm{Cu}(\mathrm{I})$ species is one of the main challenges. In most cases CuAAC is studied as a thermal reaction, although photoinduced examples have also been reported. ${ }^{3}$ It is well-known that $\mathrm{CuAAC}$ performance strongly depends on the presence of an amine. ${ }^{4}$ Concerns have been expressed about the mechanism of this reaction and many have tried to address the role of the $\mathrm{Cu}$ center and the amine ligands. ${ }^{5}$ Some reports suggest the amine is used as a base activating the alkyne for the formation of the $[\mathrm{Cu}]$-acetylide complex, ${ }^{6}$ or simply as a stabilizing ligand for $\mathrm{Cu}$ species. ${ }^{7}$ These are well-accepted mechanisms, however, they do not explain the role of the amine when the reaction is performed under heterogeneous conditions. Thus, even when catalysts have shown no leaching of $\mathrm{Cu}$ species, the amine participation on the catalytic efficiency of these materials is important. Many have suggested the amino groups help anchoring the $\mathrm{Cu}$ species to the catalyst support, thus avoiding Cu leaching. ${ }^{8}$

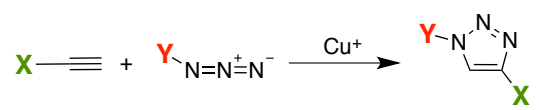

Scheme 1. Copper(I)-catalyzed azide/alkyne cycloaddition (CuAAC).

In a recent publication we reported on catalysts for photoactivated click chemistry. ${ }^{3 a}$ In particular, cupric oxide nanoparticles supported on either $\mathrm{TiO}_{2}$ or $\mathrm{Nb}_{2} \mathrm{O}_{5}$ semiconductor particles. Upon excitation of the semiconductor in the UVA region an electron is promoted from the valence band (VB) to the conduction band (CB). This electron can be transferred to a surface bound $\mathrm{CuO}$ nanoparticle, resulting in the formation of surface $\mathrm{Cu}(\mathrm{I})$ centers (Figure 1), that then can catalyze the click reaction, as illustrated in scheme 1 . The simple mechanism of Figure 1 would require ultraviolet excitation, as it is necessary to promote an electron within the semiconductor particle. Surprisingly, efficient photocatalysis also occurs upon visible light excitation in spectral regions where the light is absorbed by the small size $\mathrm{CuO}$ nanoparticles that "decorate" either $\mathrm{TiO}_{2}$ or $\mathrm{Nb}_{2} \mathrm{O}_{5}\left(\mathrm{CuO}_{\mathrm{x}} @ \mathrm{TiO}_{2}\right.$ or $\left.\mathrm{CuO}_{\mathrm{x}} @ \mathrm{Nb}_{2} \mathrm{O}_{5}\right)$. Regardless of the mechanistic details, good performance with visible light is an excellent characteristic for a photocatalyst.

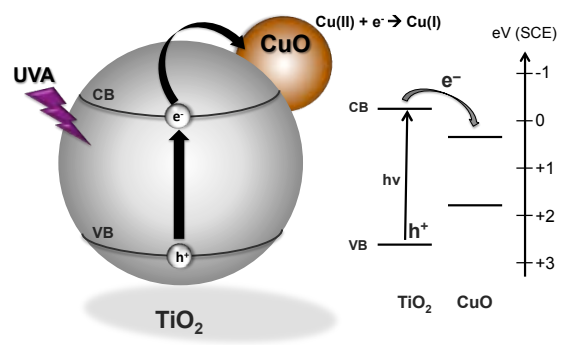

Figure 1. Proposed mechanism of electron transfer from the excited semiconductor to $\mathrm{CuO}$ nanoparticles to form catalytic $\mathrm{Cu}(\mathrm{I})$. Reprinted (adapted) with permission from (J. Am. Chem. Soc., 2016, 138, 13127-13130). Copyright (2016) American Chemical Society.

Single molecule spectroscopy (SMS) has proven a powerful tool to assist mechanistic rationalization of heterogeneous systems, such as click reactions, simply by monitoring the reaction of one molecule at the time. ${ }^{9}$ The use of Total Internal Reflection Fluorescence Microscopy (TIRFM) has helped us for example, to establish whether or not a catalytic system is homogeneous or heterogeneous, ${ }^{10}$ or to determine the true efficiency of a catalytic system. ${ }^{11}$ In this 
study, we use SMS to examine the role of an amine on the light-induced heterogeneous CuAAC. Particularly, we analyzed the behavior of $\mathrm{CuO}_{\mathrm{x}} @ \mathrm{Nb}_{2} \mathrm{O}_{5}$ when subjected to click chemistry conditions. We first proved that the process is truly heterogeneous. For this, catalytic events were detected using the same fluorescent dye combination as in earlier work, utilizing AF488 as energy donor and AF594 as acceptor (see Figure 2, top). Excitation of AF488 yields fluorescence from AF594 exclusively if the click coupling takes place, thus enabling energy transfer (Förster resonance energy transfer or FRET ${ }^{9 b}, 12$. These events can be readily discriminated using the appropriate filters (Figure 2 , bottom). In turn, the cyanine 5 amine (Cy5-A) was used to evaluate the role of the amine on the heterogeneous photocatalytic performance of $\mathrm{CuO}_{x} @ \mathrm{Nb}_{2} \mathrm{O}_{5}$ (Figure 3, top). Again the excitation of the donor (AF488) produces fluorescence from the acceptor (Cy5-A) only if they are nearneighbors as when an amino-[Cu]-acetylide complex is formed, i.e. under appropriate FRET conditions. The choice of niobium, rather than titanium oxide reflects the larger particle size of the former that facilitates co-localization of fluorescence bursts with particle location, the latter detected by optical transmission microscopy. The analysis of the fluorescence bursts observed under different conditions help us to determine the important role played by the amine: the time periods in which the $[\mathrm{Cu}]$-acetylide complex is retained on the catalyst surface are in average longer in the presence of an amine than in its absence, giving enough time for the azide reagent to encounter the catalytic site and react.

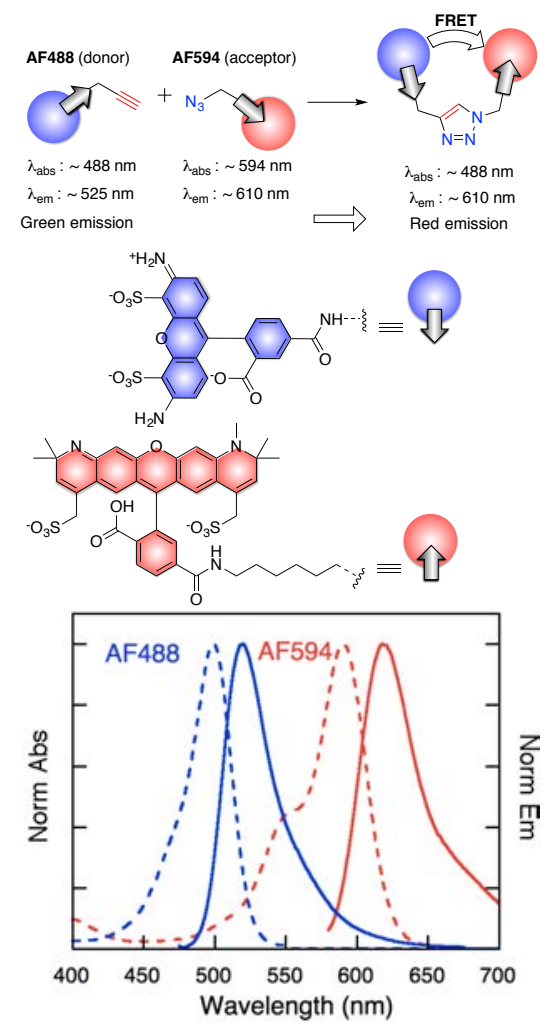

Figure 2. Top: Click reagents employed for click chemistry with fluorescent reporters. The product likely undergoes to FRET. Bottom: absorption (dash line) and emission (full line) spectra for the corresponding fluorescent reporters available online from supplier's website. ${ }^{13}$
In our previous publication ${ }^{3 a}$ we studied the photocatalytic click chemistry using $\mathrm{CuO}_{\mathbf{x}} @ \mathrm{Nb}_{2} \mathrm{O}_{5}$. Although we proved this is a very efficient photocatalyst for CuAAC, mechanistic studies using regular organic tools are rather complicated in heterogeneous systems. No leaching of $\mathrm{Cu}$ species was detected by ICP analysis, and therefore the process was considered truly heterogeneous. We also proved the reaction works much faster in the presence of an amine, e.g. triethylamine (TEA), likely involved in the rate-limiting step of the reaction. All these statements can be confirmed by a closer analysis of the intimate relation between reagents and catalytic sites: Figure 2 and 3 show the reagents selected for SMS studies of the amine role on the heterogeneous photocatalyzed CuAAC. The system was studied at the $\mathrm{pM}$ concentration range under flow conditions as depicted in Figure $\mathrm{S} 1$ utilizing $\mathrm{CuO}_{\mathrm{x}} @ \mathrm{Nb}_{2} \mathrm{O}_{5}$ (average $\mathrm{CuO}_{\mathrm{x}} \mathrm{NP}$ diameter: $39 \pm 12 \mathrm{~nm}$, Cu content: $2.51 \mathrm{wt} \%$ ) as photocatalyst. Notice that the catalyst can work under both UV or visible light illumination and therefore under the SMS conditions (488 $\mathrm{nm}$ excitation laser, see SI) both catalyst and fluorophores can be excited at once.

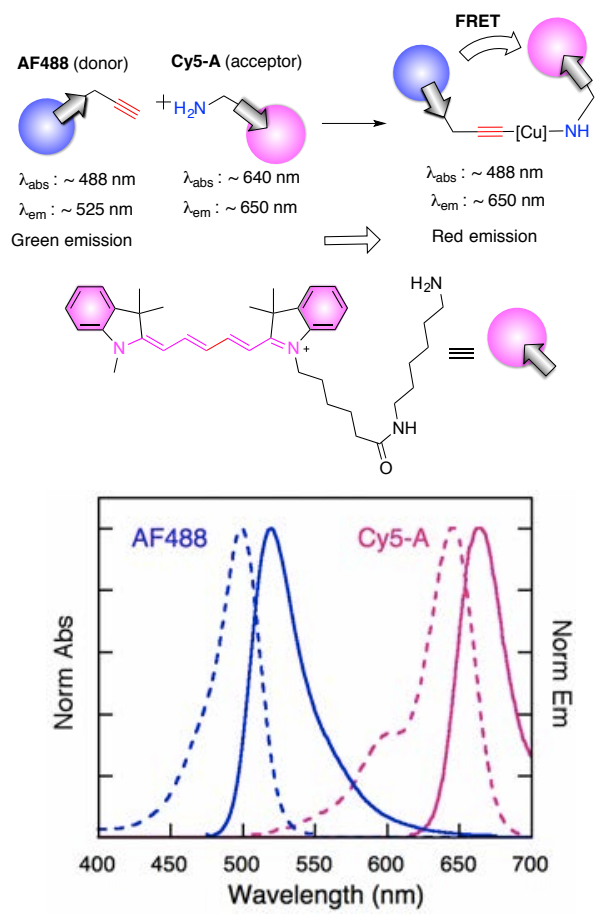

Figure 3. Top: Click reagents with fluorescent reporters employed to detect the interaction between alkyne and amine groups with the $\mathrm{Cu}$ catalytic site, abbreviated [Cu]. FRET within the complex acts as a proximity reporter. Bottom: Absorption (dash lines) and emission (bold lines) spectra of the donor (blue) and the acceptor (red) available online from supplier's website. ${ }^{13}$

We first determined the heterogeneous nature of the process as previously described. ${ }^{10}$ For this, the burst events found when only alkyne (AF488) is flowed over the catalyst were co-localized with the events originated from the occurrence of click chemistry reaction (AF488 and AF594), Figure S1. Additional observations further suggest heterogeneous catalysis (See SI).

Second, we examined the role of the amine on the heterogeneous process. Accordingly, Cy5-A has been chosen to 
follow the interaction of the amine and the alkyne at the $\mathrm{Cu}$ catalytic site. Thus, if the amine helps the formation of the $[\mathrm{Cu}]$-acetylide complex, the distance between the donor (AF488) and the acceptor (Cy5-A) would be such that FRET is possible, and one could monitor the presence of the amine (acceptor) by exciting the alkyne donor. Indeed, bright Cy5-A bursts were observed when a sample containing alkyne and amine (AF488 and Cy5-A, respectively) is excited at $488 \mathrm{~nm}$ and the emission recorded at $690 \mathrm{~nm}$ (690/70 BP, see SI). This is emission from the acceptor upon excitation of the donor probing that distance between Cy5-A and AF488 are within the requirements for FRET, i.e. formation of the amino-[Cu]-acetylide complex shown in Figure 3. Notice that under the same conditions no burst events were detected when monitoring the emission of AF488 simply by filtering the emission out using the appropriate filters (see SI). In addition, we established that the formation of this complex is co-localized with the $[\mathrm{Cu}]$-acetylide complex. For this, the conditions described for the click chemistry reaction (vide supra) were used again to co-localize bright events in the absence and in the presence of the amine (Cy5-A), Figure 4. It is important to highlight that no emission from amine was detected upon excitation at $488 \mathrm{~nm}$ when the alkyne reagent is absent.

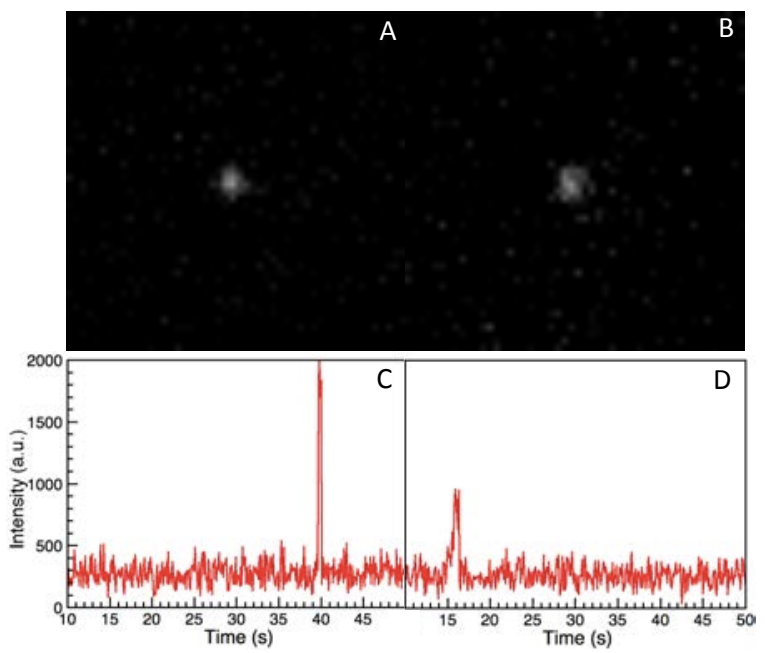

Figure 4. A) video frame showing a burst event when only alkyne reagent is flowed in the absence of amine (exc. 488 $\mathrm{nm}$ em,. 525/45 nm); B) video frame showing a burst event (located in the same 50 x 50 px area) when alkyne and amine reagents are flowed together at 100 pM concentration (exc. $488 \mathrm{~nm}$, em. $690 \mathrm{~nm}$ ); C) intensity trajectory showing burst event found in video A; and D) intensity trajectory showing burst event found in video B.

Comparative experiments in the presence of only $\mathrm{Nb}_{2} \mathrm{O}_{5}$ or $\mathrm{CuI}$ were run in order to rule out both the physical attachment of reagents to the support surface and the formation of the amino-[Cu]-acetylide complex with homogeneous $\mathrm{Cu}(\mathrm{I})$ species (see Table S2). We found that the probability for detecting FRET emission from Cy5-A in the presence of $\mathrm{Nb}_{2} \mathrm{O}_{5}$ is about $1 / 50$ comparing to the use of $\mathrm{CuO}_{\mathrm{x}} @ \mathrm{Nb}_{2} \mathrm{O}_{5}$. Indeed, the probability of the formation of homogeneous amino-[Cu]-acetylide complexes drops to almost $1 / 100$, leading to the conclusion that the vast majority $(>98 \%)$ of the burst events found in the presence of the catalyst are due to the formation of amino-[Cu]-acetylide complex on the surface of the catalyst.

Additionally, if one analyzes the traces obtained for each bright event found in different videos and measures the 'on' time ( $\tau_{\mathrm{ON}}$ ) of each burst, the distribution of the $\tau_{\mathrm{ON}}$ allows for direct examination of the role of the amine (Figure 5). Thus, the duration of each emission event for different circumstances including fluorophore photobleaching, photoblinking or product desorption from the catalyst reflects the residence times of reagents at the catalytic sites. It has been shown before that no photobleaching is found for this type of fluorophores under similar conditions. ${ }^{10,14}$ To verify that the products are able to desorb after reaction, several videos were recorded upon washing cycles over the same area; thus after recording emission events in the presence of the reagents the flow cell was rinsed with pure water and video recorded in the absence of any fluorophore. As expected, no emission events were detected under these circumstances, but the emission was easily restored once the reagents were flowed again. Hence, the "ON" times are a clear indication of the time the reagents spend at the catalytic site (Table 1), where the median has been approximated with a Gaussian expression. If one monitors the emission of AF488 (alkyne reagent) the median $\tau$ on when no amine is present (entry i) is $0.96 \mathrm{~s}$, but longer $\tau$ ON are found when in the presence of amines (1.30 s and $1.19 \mathrm{~s}$ for TEA and Cy5-A, respectively). More interestingly, if only the amino- $[\mathrm{Cu}]$-acetylide complex is monitored (entry iv), the $\tau_{\mathrm{ON}}$ increases to $1.71 \mathrm{~s}$. Notice that the $\tau_{O N}$ for the click reaction (entry v, Figure S4) is around $2.09 \mathrm{~s}$, similar to values previously found.10 Consequently, it is suggested that the amine prolongs the time that the alkyne spends on the catalyst surface, thus increasing the probability of encounters with the azide.
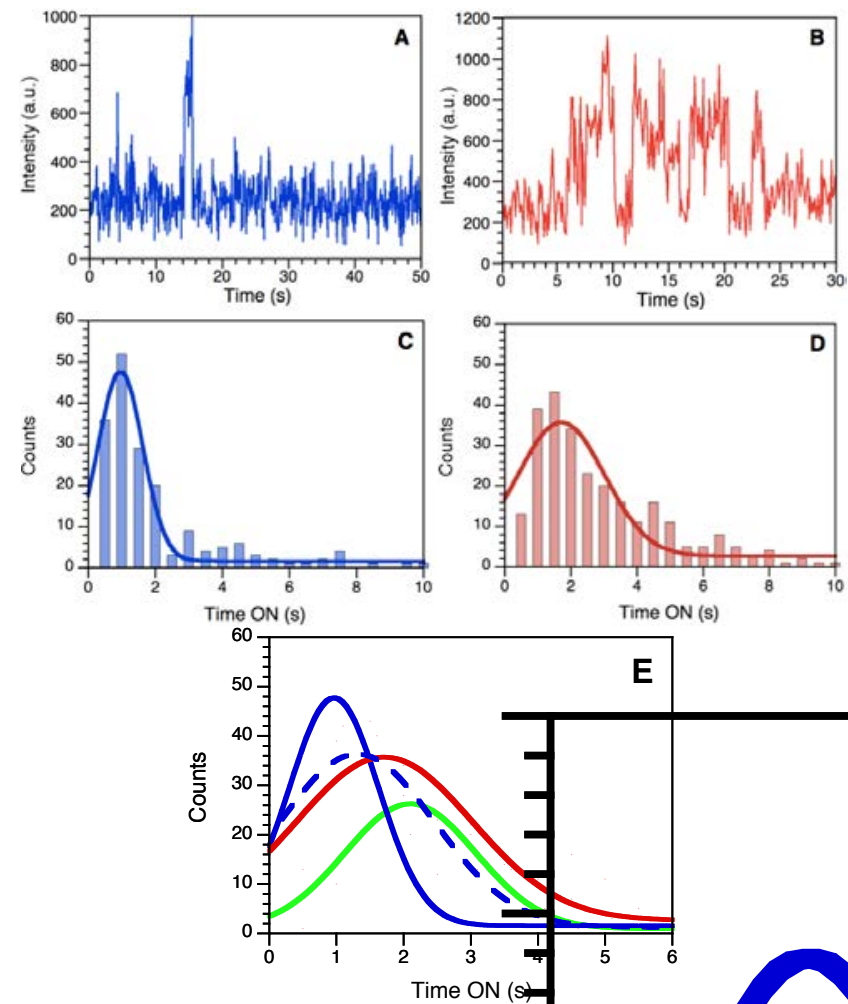
Figure 5. Selected intensity trajectories that illustrate well the observation of the burst events of different duration ( $t_{0 N}$ ) (top) and Ton distribution in a total of 12 videos (bottom) for A-C) AF488 (table 1, entry i) and B-D) AF488 plus Cy5-A (table 1, entry iii). E) Ton distribution overlay helps the eye see differences between AF488 (full blue line), AF488 plus TEA (dash blue line), AF488 plus Cy5-A (red line) and AF488 plus AF594 plus TEA (green line).

Interestingly the green curve in Figure 5E, corresponding to the full reactive system suggests that the product is not significantly retained in the catalytic site (notice small displacement between green and red curves). This is a desirable property, as the catalytic site becomes available for reaction as soon as the product leaves. Beyond the interpretation of heterogeneous CuAAC chemistry, histogram based functions, as in Figure 5E are a powerful tool to understand the role of co-catalysts in heterogeneous reactions.

Table 1. Median ' $O N$ ' times for different systems in the presence and absence of an amine

\begin{tabular}{lllll}
\hline & Reagents & $\begin{array}{l}\text { Exc } \\
(\mathrm{nm})\end{array}$ & $\begin{array}{l}\text { Em } \\
(\mathrm{nm})\end{array}$ & Time ON (s) \\
\hline i & AF488 & 488 & 525 & 0.96 \\
ii & AF488 + Cy5-A & 488 & 525 & 1.19 \\
iii & AF488 + Cy5-A & 488 & 690 & 1.71 \\
iv & AF488 + TEA & 488 & 525 & 1.30 \\
v & Cy5-A & 635 & 690 & 1.22 \\
vi & AF488+AF594+TEA & 488 & 610 & 2.09 \\
\hline
\end{tabular}

A careful analysis of the fluorescence trajectories found for entry iii in table 1 show around $1 \%$ of multiple $0 N$-levels (Figure 6). There are two situations possible for multiple on-level events to occur: (i) two amino-[Cu]-acetylide complexes are formed within the monitored area or, (ii) an (amino) $2-[\mathrm{Cu}]$-acetylide type of complex formed (Figure S6). Since no multiple on-levels events were found when monitoring the alkyne emission $(525 \mathrm{~nm})$ or click reaction emission $(645 \mathrm{~nm})$, this could favor the presence of a multiple amine complexes as suggested in Figure S6.
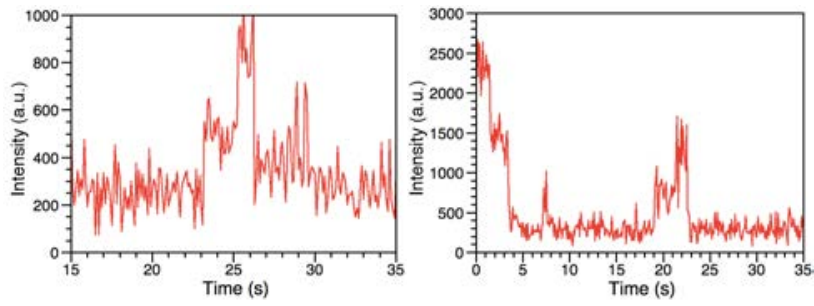

Figure 6. Multiple ON-level events found under conditions of table 1, entry iii. Capture rate is 10 frames per second giving $100 \mathrm{~ms}$ temporal resolution. Events occurring stepwise within $100 \mathrm{~ms}$ appear as single events.

\section{CONCLUSIONS}

SMS studies were used to determine the role of the amine on the heterogeneous photocatalyzed CuAAC. We estab- lished that $\mathrm{CuO}_{\mathrm{x}} @ \mathrm{Nb}_{2} \mathrm{O}_{5}$ catalyzes this type of cycloadditions through a heterogeneous process. Notably, the amine plays an important role in the complexation between the alkyne and the copper active site on the catalyst surface we have found that the $[\mathrm{Cu}]$-acetylide complex can be retained on the catalyst surface longer in the presence of an amine, thus providing extended opportunities for the azide to come in and react. Indeed, the amine seems to be part of this complex as we proved the amine and alkyne dye moieties are in close proximity, enough to undergo FRET under excitation of the alkyne dye. This is in agreement with our previous findings in which the amine seems to facilitate the reaction decreasing drastically the reaction times. ${ }^{3 a}$ Thus, the longer the time of the $[\mathrm{Cu}]$-acetylide complex lives, the higher the probability of encounter and reaction with the azide. In complex events requiring alkyne, [Cu], amine and azide and occurring in the sub-nanomolar range, pre association seems essential and any effect (i.e., amine) that prolong the association time can play a critical role in the reaction. This is to the best of our knowledge the first time the amine role is rationalized in heterogeneous photocatalytic click chemistry.

\section{Supporting Information}

Details on the instrumentation used, SMS level experiments, and complementary experiments to prove heterogeneous catalysis can be found in the Supporting Information. The Supporting Information is available free of charge on the ACS Publications website.

\section{AUTHOR INFORMATION}

\section{Corresponding Author}

*J.C.S.: titoscaiano@mac.com

*A.E.L.: anabel.lanterna@icloud.com

ORCID

Juan C. Scaiano: 0000-0002-4838-7123

Anabel E. Lanterna: 0000-0002-6743-0940

Bowen Wang: 0000-0002-3398-8348

\section{Notes}

The authors declare no competing financial interest.

\section{ACKNOWLEDGMENT}

We thank the Natural Sciences and Engineering Research Council, the Canada Foundation for Innovation, and the Canada Research Chairs Program. The authors would like to thank Dr. Narayan Bulusu for kindly providing his help during data analysis.

\section{REFERENCES}

1. Rostovtsev, V. V.; Green, L. G.; Fokin, V. V.; Sharpless, K. B., A stepwise Huisgen cycloaddition process: Copper(I)-catalyzed regioselective "ligation" of azides and terminal alkynes. Angew. Chem. Int. Ed. 2002, 41 (14), 2596-2599.

2. Tornøe, C. W.; Christensen, C.; Meldal, M., Peptidotriazoles on solid phase: [1,2,3]-triazoles by regiospecific copper(I)-catalyzed 1,3-dipolar cycloadditions of terminal alkynes to azides. J. Org. Chem. 2002, 67 (9), 3057-3064.

3. (a) Wang, B.; Durantini, J.; Nie, J.; Lanterna, A. E.; Scaiano, J. C., Heterogeneous Photocatalytic Click Chemistry. J. Am. Chem. Soc. 2016, 138 (40), 13127-13130; (b) Arumugam, S.; Orski, S. V.; Mbua, N. E.; McNitt, C.; Boons, G. J.; Locklin, J.; Popik, V. V., Photo-click chemistry strategies for spatiotemporal control of 
metal-free ligation, labeling, and surface derivatization. Pure Appl. Chem. 2013, 85 (7), 1499-1513; (c) Orski, S. V.; Poloukhtine, A. A.; Arumugam, S.; Mao, L. D.; Popik, V. V.; Locklin, J., High Density Orthogonal Surface Immobilization via Photoactivated CopperFree Click Chemistry. J. Am. Chem. Soc. 2010, 132 (32), 11024 11026.

4. Haldon, E.; Nicasio, M. C.; Perez, P. J., Copper-catalysed azide-alkyne cycloadditions (CuAAC): an update. Org Biomol Chem 2015, 13 (37), 9528-9550.

5. (a) Ahlquist, M. r.; Fokin, V., Enhanced Reactivity of Dinuclear Copper(I) Acetylides in Dipolar Cycloadditions Organomet. 2007, 26, 4389-4391; (b) Hein, J. E.; Fokin, V. V., Copper-catalyzed azide-alkyne cycloaddition (CuAAC) and beyond: new reactivity of copper(I) acetylides. Chem. Soc. Rev. 2010, 39 (4), 1302-1315; (c) Rodionov, V. O.; Fokin, V. V.; Finn, M. $\mathrm{G}$., Mechanism of the ligand-free $\mathrm{Cu}$-I-catalyzed azide-alkyne cycloaddition reaction. Angew. Chem. Int. Ed. 2005, 44 (15), 2210 2215; (d) Worrell, B. T.; Malik, J. A.; Fokin, V. V., Direct Evidence of a Dinuclear Copper Intermediate in Cu(I)-Catalyzed Azide-Alkyne Cycloadditions. Science 2013, 340 (6131), 457-460; (e) Wang, C. L.; Ikhlef, D.; Kahlal, S.; Saillard, J. Y.; Astruc, D., Metal-catalyzed azide-alkyne "click" reactions: Mechanistic overview and recent trends. Coord. Chem. Rev. 2016, 316, 1-20.

6. (a) Alonso, F.; Moglie, Y.; Radivoy, G., Copper Nanoparticles in Click Chemistry. Acc. Chem. Res. 2015, 48 (9), 2516-2528; (b) Hein, J.; Fokin, V., Copper-catalyzed azide-alkyne cycloaddition (CuAAC) and beyond: new reactivity of copper(I) acetylides. Chem. Soc. Rev. 2010, 39 (4), 1302-1315.

7. (a) Rodionov, V. O.; Presolski, S. I.; Gardinier, S.; Lim, Y. H.; Finn, M. G., Benzimidazole and related Ligands for $\mathrm{Cu}-$ catalyzed azide-alkyne cycloaddition. J. Am. Chem. Soc. 2007, 129 (42), 12696-12704; (b) Rodionov, V. O.; Presolski, S. I.; Diaz, D. D.; Fokin, V. V.; Finn, M. G., Ligand-accelerated $\mathrm{Cu}$-catalyzed azide- alkyne cycloaddition: A mechanistic report. J. Am. Chem. Soc. 2007, 129 (42), 12705-12712.

8. Chassaing, S.; Beneteau, V.; Pale, P., When CuAAC 'Click Chemistry' goes heterogeneous. Cat. Sci. Technol. 2016, 6 (4), 923-957.

9. (a) Wang, B. W.; Durantini, J.; Decan, M. R.; Nie, J.; Lanterna, A. E.; Scaiano, J. C., From the molecule to the mole: improving heterogeneous copper catalyzed click chemistry using single molecule spectroscopy. Chem. Commun. 2017, 53 (2), 328331; (b) Scaiano, J. C.; Lanterna, A. E., Is Single-Molecule Fluorescence Spectroscopy Ready To Join the Organic Chemistry Toolkit? A Test Case Involving Click Chemistry. J. Org. Chem. 2017, 82 (10), 5011-5019; (c) Buurmans, I. L. C.; Weckhuysen, B. M., Heterogeneities of individual catalyst particles in space and time as monitored by spectroscopy. Nature Chem. 2012, 4, 873886.

10. Decan, M. R.; Impellizzeri, S.; Marin, M. L.; Scaiano, J. C., Copper nanoparticle heterogeneous catalytic 'click' cycloaddition confirmed by single-molecule spectroscopy. Nat. Commun. 2014, $5,4612-4617$

11. Decan, M. R.; Scaiano, J. C., Study of Single Catalytic Events at Copper-in-Charcoal: Localization of Click Activity Through Subdiffraction Observation of Single Catalytic Events. J. Phys. Chem. Lett. 2015, 6, 4049-4053.

12. (a) Hohlbein, J.; Craggs, T.; Cordes, T., Alternating-laser excitation: single-molecule FRET and beyond. Chem. Soc. Rev. 2014, 43 (4), 1156-1171; (b) Turro, N. J. Ramamurthy, V.; Scaiano, J. C. , Modern Molecular Photochemistry of Organic Molecules. University Science Publishers: New York, N.Y.: 2010.

13. https://www.thermofisher.com.

14. Chen, P.; Zhou, X.; Shen, H.; Andoy, N. M.; Choudhary, E.; Han, K.-S.; Liu, G.; Meng, W., Single-molecule fluorescence imaging of nanocatalytic processes. Chem. Soc. Rev. 2010, 39, 4560-4570. 
Insert Table of Contents artwork here

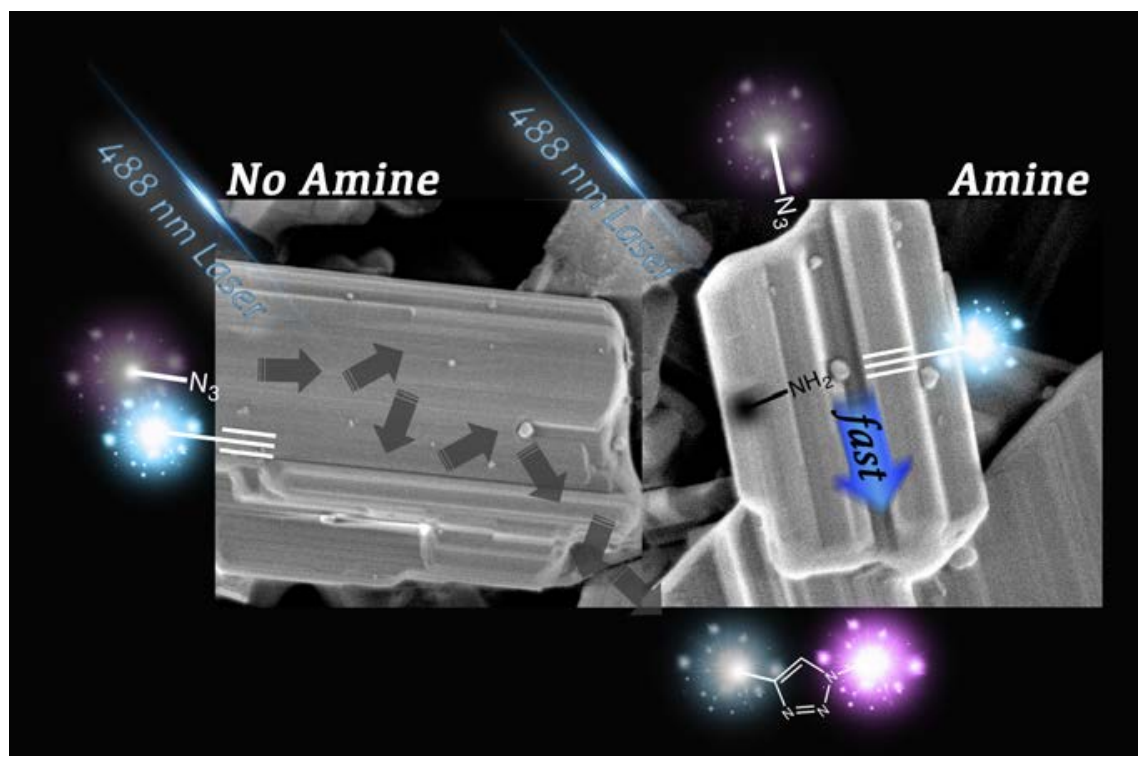

